\title{
ARC: an Achromatic Rotation-shearing Coronagraph
}

\author{
C. Aime ${ }^{1}$, G. Ricort ${ }^{1}$, A. Carlotti ${ }^{1,2}$, Y. Rabbia ${ }^{1}$, and J. Gay ${ }^{1}$ \\ 1 UMR 6525 H. Fizeau, Université de Nice-Sophia Antipolis, Centre National de la Recherche Scientifique, \\ Observatoire de la Côte d'Azur, Parc Valrose, 06108 Nice, France \\ e-mail: [claude.aime; gilbert.ricort;alexis.carlotti]@unice.fr; [yves.rabbia; jean.gay]@obs-azur.fr \\ 2 Princeton University, Princeton, NJ, 08544, USA \\ e-mail: acarlott@princeton.edu
}

Received 20 July 2009 / Accepted 1 March 2010

\section{ABSTRACT}

\begin{abstract}
Context. Because of the larger size of future telescopes, the star leakage effect due to the finite diameter of the stars will become a major problem for coronagraphs with very small inner working angles (IWA).

Aims. To reduce this star leakage, we propose a new instrumental concept, the Achromatic Rotation-shearing Coronagraph (ARC), that provides a variable IWA that is easily tunable to ensure the observation of Solar-like systems with an extremely large telescope. Methods. The ARC belongs to the nuller class of coronagraphs. It takes advantage of the achromatic dark output of a Mach-Zehnder interferometer (MZI). Field rotators set in the arms of the MZI rotate the $\pi$-phase shifted images of the MZI by an angle $\psi$. An on-axis point source (the star) is nulled as twin-images of an off-axis point source (the planet) are formed. For $\psi=\pi$, the ARC is equivalent to the achromatic interfero-coronagraph. For small $\psi$, the twin images of the off-axis source are formed close to each another, and eventually nulled if too close. In this way, the ARC can get rid of star leakage while optimizing the transmission of the planet. We describe the compensation of polarization effects induced by field rotators. An application to diluted apertures is shortly presented. Results. We perform the mathematical analysis of the technique using Cartesian and polar coordinates, and assuming that the system is optically perfect. Analytic expressions are given for the leakage in both the focal and aperture planes. Simplified expressions for the contrast and signal-to-noise ratio (SNR) in the focal plane are given for a circular aperture, explicitly showing that the level of star leakage decreases as $\sin ^{2}(\psi / 2)$, while the SNR increases as the inverse of $\sin (\psi / 2)$.

Conclusions. The ARC is appropriate for the detection of exoplanets and is not intended to provide images of the environment of stars.
\end{abstract}

Key words. instrumentation: high angular resolution - techniques: high angular resolution - techniques: interferometric

\section{Introduction}

Since 2004, a dozen planets have been observed directly thanks to improved techniques of adaptive optics and the postprocessing of the images (Chauvin et al. 2004; Marois et al. 2008). These planets are mostly giant planets situated tens of astronomical units from their parent stars ${ }^{1}$. However, the direct observation of planets similar to those of our solar system will require the use of coronagraphs.

These devices aim to remove the contribution of the star from the image plane so as to improve the detectability of the planet. The extinction of the diffracted star light is never complete and some residual energy remains in the image plane. A first figure of merit for these systems is the rejection, or its inverse, the nulling depth or extinction. Rejection is defined as the ratio of the collected energy to the residual energy. Required rejections depend on the science target. For an exo-Earth, it is of the order of $10^{6}$ at $10 \mu \mathrm{m}$, and might reach $10^{10}$ in the visible part of the spectrum (Ridgway 2004)

Another figure of merit, the inner working angle (IWA), determines how close to the star the observation of an exoplanet can be made. We must take into account the star leakage of the central star, which, although the star is not resolved, will dramatically hamper the efficiency of the coronagraph. Since exoplanets are faint sources, the ultimate figure of merit is the

\footnotetext{
${ }^{1}$ See website http://exoplanet. eu, for example.
}

signal-to-noise ratio (SNR) of the experiment. Reliable detection requires the collection of as many photons as possible and the coronagraph to be achromatic over a wide spectral bandwidth.

Devising instrumental concepts for exoplanet detection, for which renewed interest started more than a decade ago, remains an active field and various approaches have been considered besides the historical coronagraph of Lyot $(1932,1939)$ and the nulling interferometer proposed by Bracewell (1978). A collection of papers describing a few of these systems, including external occulters, Bracewell-like interferometers, apodized apertures, phase masks and band-limited coronagraphs can be found for example in Aime (2007). Recent promising advances have been achieved with optical vortex coronagraphs (Swartzlander et al. 2008; Mawet et al. 2010).

The achromatic interfero coronagraph (AIC) of Gay \& Rabbia (1996), detailed in Gay et al. (1997) and Baudoz et al. (2000a,b), takes a particular place among these coronagraphs. In principle, it belongs to the class of nuller coronagraphs. When working with a single aperture, the incoming wave is divided into two components, one of which undergoes a $\pi$-phase shift because of the focus crossing property and a 180 degrees field rotation. For an on-axis source, this yields a null in the image plane when the two sub-waves are recombined. The AIC has several interesting features: its $\pi$ phase shift is fully achromatic, and it is the most insensitive to the aperture shape (being usable with a central obstruction) providing the narrowest IWA of 
all coronagraphs (smaller than $\lambda / D$ ), as pointed out by Guyon (2007). This enables an area very close to the star to be explored. This remarkable property has two drawbacks, a high sensitivity to pointing errors, which requires a perfect pointing system, and a high sensitivity to the effective angular size $\Theta$ of the star, a problem that worsens as the telescope diameter increases. To improve the detection in the presence of star leakage, Tavrov et al. (2008) proposed using a nulling out-of-plane coronagraph with two common-path interferometers in tandem. We note that this is a common problem for many coronagraphs, and for the same objective of reducing star leakage the use of eight octants phase masks was proposed by Murakami et al. (2008) instead of the original four quadrant phase mask of Rouan et al. (2000).

The AIC duplicates the images in the focal plane. This is a problem when studying a complex stellar environment, but not for the imaging of an exoplanet. Since the duplication provides two symmetrical images, there is also a quadrant uncertainty on the relative position of the star and the planet. This can be solved with the present proposal of an Achromatic Rotationshearing Coronagraph (ARC), in a way similar to that of the Absolute Position Interfero Coronagraph (APIC) proposed recently by Allouche et al. (2009), since it introduces a variable separation between the two focal images of the planet. However, ARC differs from APIC in terms of set-up and implementation, which we comment more later in the paper.

Apart from the interest in removing the indetermination of quadrant, the main advantage of the ARC is certainly its capability to reduce star leakage. In this study our motivation is similar to that of Tavrov et al. (2008) who used a 3D common-path Sagnac interferometer. As we demonstrate, the ARC concept has certain advantages provided that the aperture is invariant under a rotation by an angle $\psi$. A full circular aperture (with or without a circular central obstruction) verifies this condition for any $\psi$ value. A few other apertures (square, hexagon, ...) would satisfy this condition for particular values of $\psi$. This property extends to diluted apertures for particular configurations, as discussed at the end of this paper.

The paper is organized as follows. The principle of the ARC and two possible assemblies are described in Sect. 2. The mathematical descriptions of the amplitudes and intensities in the focal plane and aperture plane are given in Sect. 3. The problem of star leakage is treated in Sect. 4, where the result is used in considering both contrast and SNR. Application of the ARC to diluted apertures is briefly introduced in Sect. 5 and conclusions are drawn in Sect. 6.

\section{Basic principle of ARC}

As already indicated, the ARC belongs to the same class of coronagraphs as AIC and behaves like a nulling system. While AIC is a modification of the Michelson interferometer (MI), ARC uses the principle of the Mach-Zehnder interferometer (MZI). They both perform a null of the star by superimposing rotated and achromatically $\pi$ dephased amplitude distributions over the aperture. For AIC, the rotation is fixed to 180 degrees, while it is tunable for ARC. To achieve the nulling, ARC directly uses the dark fringe of the MZI, while AIC must darken the white fringe of the MI by inserting an achromatic $\pi$ phase shift in one arm. This dark fringe property of the MZI of behaving like a subtractive device was applied in Aime et al. (2007) and Carlotti et al. (2008, 2009). For an on-axis point source the achromatic nulling property for AIC and ARC is similar. For an off-axis source, they both produce twin images, at 180 degrees for AIC but at a tunable angle for ARC. This is possible by means of a field

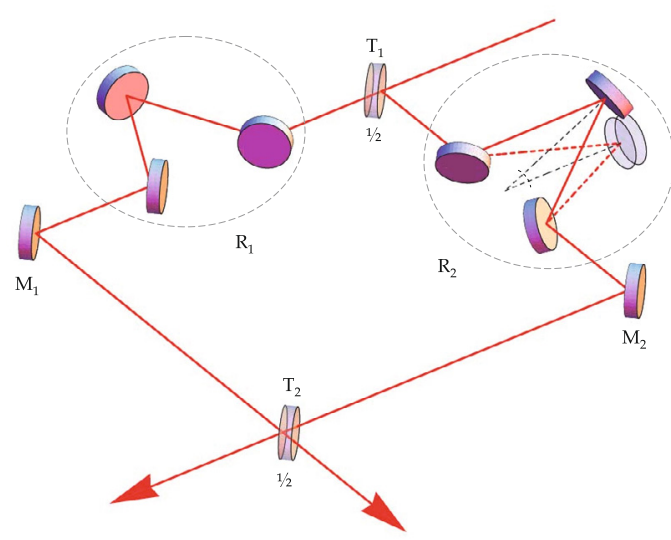

Fig. 1. Assembly of the MZI interferometer called ARC1 in the paper. It is a classical MZI in which two field rotators $R_{1}$ and $R_{2}$ have been included in the arms. A rotation of $R_{2}$ is schematized there.

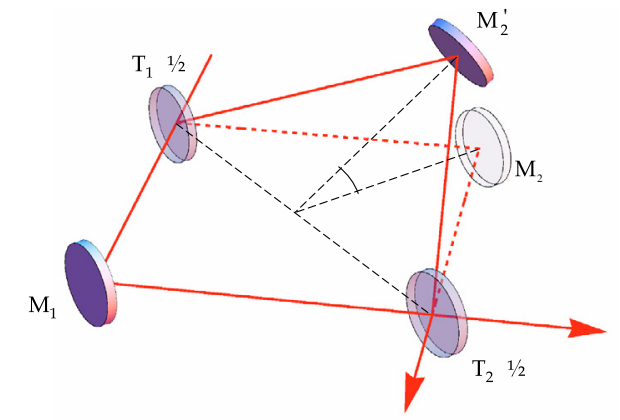

Fig. 2. Assembly of the MZI interferometer called ARC2 in the paper. The field rotation is obtained by folding one arm of the MZI. The rotation of $\mathrm{M}_{2}$ schematized here implies a corresponding rotations of the beamsplitters $\mathrm{T}_{1}$ and $\mathrm{T}_{2}$.

rotation, a feature also proposed in APIC and in Strojnik \& Paez (2007).

In Figs. 1 and 2 we indicate for ARC two possible optical configurations by which the MZI becomes a rotation shearing interferometer. In the figures we show neither the parallel beam entering ARC from the telescope, nor at the dark output of the MZI (output pupil), the beam focused on the image plane. The system shown in Fig. 1 departs from the classical MZI, simply by the insertion of two field rotators, denoted $R_{1}$ and $R_{2}$, one in each arm. The MZI is represented instead in its classical planar and rectangular layout using two mirrors $\left(\mathrm{M}_{1}\right.$ and $\left.\mathrm{M}_{2}\right)$ and two beam-splitters $\left(T_{1}\right.$ and $\left.T_{2}\right)$, in which it is mandatory to have two identical arms so as to accurately balance the optical paths before recombination. In the figure, we show how both field rotators are used to maintain the layout as symmetrically as possible. However, not shown is the way in which we cope with successive reflections (same number of turns, respectively, to the left and to the right in each arm) to meet the recommendation pertaining to polarizations, given in Traub (1988). We note that the global field rotation by an angle $\psi$ is thus obtained by two rotations of angle $\psi / 2$, one in each arm and of opposite signs. In the subtractive output of the MZI, the null will be obtained for an onaxis point-source regardless of the value of $\psi$ because the image of the star will only rotate without lateral motion. In contrast, for the twin-images of the planet, as $\psi$ increases, the separation increases and the null progressively disappears. We refer to this set-up as ARC1. 
The system shown in Fig. 2 only uses the mirrors and beamsplitters of the original MZI but in a non-planar configuration. The MZI is folded by a small angle, the two beam-splitters being used as pivot hinges of the rotation. The first beamsplitter $\mathrm{T}_{1}$ directs the light above the optical table towards the new position $\mathrm{M}_{2}^{\prime}$ of $\mathrm{M}_{2}$, then $\mathrm{M}_{2}^{\prime}$ redirects the light towards $\mathrm{T}_{2}$. One can consider $\mathrm{T}_{1} \mathrm{M}_{2} \mathrm{~T}_{2}$ to be rotated as whole into $\mathrm{T}_{1} \mathrm{M}_{2}^{\prime} \mathrm{T}_{2}$. In that set-up, the system of the two beam-splitters and the mirror serves as the field rotator. This layout requires fewer optical elements than the former and may appear more elegant, but the beamsplitters are used with non-orthogonal angles, which prevents for example the use of cubes as beamsplitters and is likely to introduce spurious polarization effects. We refer to this assembly as ARC2.

The ARC2 configuration is similar to the 3D common-path Sagnac interferometer of Tavrov et al. (2008), while ARC1 is similar to APIC, which also uses a MZI. The devices ARC1 and APIC differ in that APIC has a system of two cylindrical lenses inserted in one arm of the MZI. The authors explain that this system is used as a substitute for the cat's eye used in AIC to obtain a $\pi$ phase shift, which is able to produce a variable angle rotation as in ARC. As described above, the focus $\pi$ phasing shifting property is not necessary since the MZI inherently provides the $\pi$ phase difference at its subtractive output. Since it is mandatory to maintain an accurate balance between the optical paths, the APIC set-up also requires some kind of delay line to be inserted in the MZI. In the following, ARC indicates ARC1.

\section{Mathematical description of the experiment}

The use of rotation inside the MZI produces a rather complex superimposition of the images. To perform a detailed mathematical analysis of ARC, we use both Cartesian and polar coordinates. The Cartesian system is well adapted to describing the distribution of amplitude and intensities in the focal plane, the polar system is more convenient for working in the aperture plane. The polar system is also used to quantify the spatial transmission of the coronagraph and the leakage effect induced by a star of finite diameter. If not otherwise specified, the mathematical formalism used is one of Fourier optics (see for example Goodman 1985). The algebraic derivations are applicable as much as possible to the diluted array mode of operation.

\subsection{Basic equations in Cartesian coordinates: focal plane images.}

We define $\xi$ and $\eta$ to be the Cartesian coordinates in the aperture plane, and $P(\xi, \eta)$ the function that describes the telescope aperture. We define $\alpha$ and $\beta$ to be the position angles on the sky. These coordinates are also used to describe a position in the focal plane. Denoting as $x$ and $y$ the coordinates in the focal plane, the angular conversion is readily obtained as $\alpha=x / F$ and $\beta=y / F$, where $F$ is the focal length of the telescope. Image inversion is often neglected to avoid an unhelpful change in the coordinates's orientation from the sky to the image plane.

For a plane wave incident normal to the aperture plane of unit intensity, the amplitude in the image plane is the response of the telescope to an on-axis point source and can be written as

$A(\alpha, \beta)=\frac{1}{\lambda} \hat{P}\left(\frac{\alpha}{\lambda}, \frac{\beta}{\lambda}\right)$

where the symbol $\hat{P}$ stands for the 2D Fourier transform of $P$.

As a MZI, ARC splits and recombines the beams by means of its two beamsplitters. Because of the field rotators, the respective complex amplitude distributions in the image plane are rotated one with respect to the other. The combination of refraction and reflection indices by the beamsplitters and its effects were described by Carlotti et al. (2008). As discussed there, a MZI can be a perfect subtractor, if the beam splitter coefficients $r_{B S}$ and $t_{B S}$ are equal. This is indeed critical and a fine tuning of the effective transmission in each arm of the MZI (and over the whole spectral bandwidth of work) will have to be considered, as proposed in Carlotti et al. (2009).

Another problem inherent to rotation-shearing interferometers is polarization. As explained in detail by Roddier et al. (1978) in another context, "rotation-shearing interferometers have a severe drawback: when unpolarized light is used at the interferometer input, the state of polarization of the two beams at the output do not in general match... the net result is that good fringe contrast is obtained only for small angles of rotation". In the case of ARC, the effective rejection of the coronagraph may be strongly reduced. Roddier et al. (1978) proposed a very elegant solution to that problem, using neither a polarizer nor an analyzer, which was applied to a rotation shearing interferometer using roof-prisms set in a Michelson-like configuration. It consisted of cementing to the entrance face of the roof-prisms an appropriate phase plate with principal axes parallel and perpendicular to the prism edges. In their experiment, they used quartz phase plates, constructed in the optical workshop of the Nice Observatory, to produce the retardation angle of $53^{\circ}$ required by their experiment. Their results showed a fringe visibility fairly constant and maximal for any angle of rotation. The experiment was used to observe Betelgeuse (Roddier \& Roddier 1983; Roddier et al. 1986). The transposition to the ARC configuration using either Dove prisms or a system of mirrors remains to be performed, and the question remains whether this phase compensation will be able to produce the ultra deep nulling required for exoplanet detection. Dove Prisms probably lead to a result more directly comparable to that of Roddier et al. (1978), while for the mirrors, the system compensation should take into account the elliptic polarization induced by the metallic surface. Here, we do not consider the problem further, knowing that it will require theoretical as well as experimental new developments.

Rotating the wavefront at the aperture by an angle $\psi$, the resulting amplitude simply undergoes a change in coordinates, by applying a rotation matrix $\left(\begin{array}{cc}\cos \psi & \sin \psi \\ -\sin \psi & \cos \psi\end{array}\right)$ to the vector $(\alpha, \beta)$.

After a rotation of $\psi, A(\alpha, \beta)$ will be noted as the function

$A_{\psi}(\alpha, \beta)=A(\alpha \cos (\psi)+\beta \sin (\psi),-\alpha \sin (\psi)+\beta \cos (\psi))$

A point source at the position $\left(\alpha_{0}, \beta_{0}\right)$ on the sky produces the response $A_{\psi}\left(\alpha-\alpha_{0}, \beta-\beta_{0}\right)$ in the focal plane.

As above mentioned the global rotation by $\psi$ consists of combining two half-rotations (by $+\psi / 2$ in arm 1 and by $-\psi / 2$ in arm 2). This fortunately simplifies the mathematical formalism. We assume perfect optics, so that for each beamsplitter the reflection and transmission coefficients for the amplitudes are equal, i.e. $r_{B S}=t_{B S}=\frac{\sqrt{2}}{2}$. Since each beam undergoes two reflections and/or transmissions, the amplitudes in the additive and subtractive outputs are affected by a transmission 1/2. Amplitudes at the outputs are written as

$A_{\psi}^{ \pm}(\alpha, \beta)=\frac{1}{2}\left(A_{\psi / 2}\left(\alpha-\alpha_{0}, \beta-\beta_{0}\right) \pm A_{-\psi / 2}\left(\alpha-\alpha_{0}, \beta-\beta_{0}\right)\right)$,

where the symbol \pm in this equation is equal to + for the additive output $A_{\psi}^{+}(\alpha, \beta)$ and equal to - for the subtractive output 

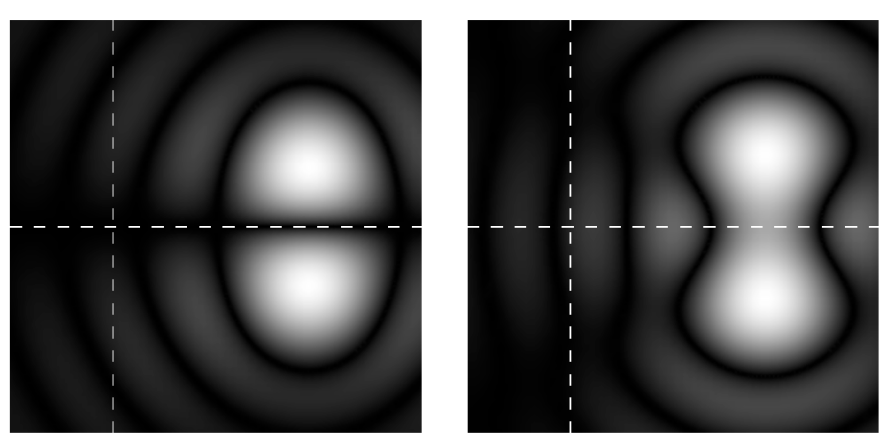

Fig. 3. Intensities observed in the subtractive (left) and additive (right) outputs as given in Eq. (5). The figure is drawn for a point source situated at $\rho_{0}=3 \lambda / D$ in the $\alpha$ direction, and the angle of rotation is $\psi=\pi / 5$.

$A_{\psi}^{-}(\alpha, \beta)$, and where the $\pi$ phase difference is applied to path 2 . Intensities are simply the squared modulus of these expressions.

For an on-axis source, the amplitude distributions $A_{\psi / 2}(\alpha, \beta)$ and $A_{-\psi / 2}(\alpha, \beta)$ during the rotation remain superimposed. Provided that the aperture is unchanged after rotation by $\psi$, these functions are identical and the starlight is fully suppressed.

For an off-axis source, the rotation moves the centers of the twin-images along a circular path of radius $\rho_{0}=\left(\alpha_{0}^{2}+\beta_{0}^{2}\right)^{1 / 2}$. The greater the distance $\rho_{0}$, the wider the separation between the twin images. The linear distance between their center is $2 \rho_{0} \sin (\psi / 2)$, the maximum of which is $2 \rho_{0}$ for $\theta=\pi$ corresponding to the AIC configuration. With the two half rotations $\pm \psi / 2$ used, the displacement is normal to the direction of the off-axis source projected over the aperture.

The reader should note that the on-axis point source is ideally an unresolved point-like star, while the off-axis point source is the planet. Because of pointing errors, the star may become a slightly off-axis source. Moreover, the off-axis equations will be needed to compute the star leakage of the star, even if it is perfectly centered on the optical axis of the experiment.

\subsubsection{Simplified example of a circular aperture}

We consider the simple example of an unresolved on-axis star and an exoplanet located at the angular distance $\rho_{0}$ in the direction described by $\alpha$. Observations are performed using a circular aperture of diameter $D$, and Eq. (1) then becomes

$A_{\odot}(\alpha, \beta)=\frac{D J_{1}(\pi D \rho / \lambda)}{2 \rho}, \quad$ with $\quad \rho=\left(\alpha^{2}+\beta^{2}\right)^{1 / 2}$

and the intensities in the additive and subtractive (coronagraphic) outputs can be synthetically expressed by

$$
\begin{aligned}
I_{\odot}^{ \pm}(\alpha, \beta)=\frac{1}{4} & \mid A_{\odot}\left(\alpha-\rho_{0} \cos \left(\frac{\psi}{2}\right), \beta-\rho_{0} \sin \left(\frac{\psi}{2}\right)\right) \\
& \pm\left. A_{\odot}\left(\alpha-\rho_{0} \cos \left(\frac{\psi}{2}\right), \beta+\rho_{0} \sin \left(\frac{\psi}{2}\right)\right)\right|^{2},
\end{aligned}
$$

where we use the same convention for \pm as in Eq. (3). The rotated images of the planet are centered on the positions $\alpha=$ $\rho_{0} \cos (\psi / 2)$ and $\left.\beta= \pm \rho_{0} \sin (\psi / 2)\right\}$. The observed intensities slightly differ between the two outputs, mainly for close-by twin images, as shown in the illustrative example of Fig. 3. Parameters are chosen there to enhance differences between additive and subtractive outputs. We note that the additive output is blinded out by the star image, suppressed in this representation. The images for $I_{\odot}^{-}(\alpha, \beta)$ are similar to those given in Allouche et al. (2009). Since the starlight is rejected in the additive output, only $I_{\psi}^{-}(\alpha, \beta)$ is of interest here. We note that the light of the planet present in the additive output might be partially recovered if a second coronagraphic stage is used, but this output will preferably be used to improve the quality of the coronagraph, for example to feed a fine pointing system, or for optimal wavefront correction (see for example Labeyrie 2006).

\subsection{Aperture plane: basic equations in polar coordinates}

To describe the amplitudes in the aperture plane, it is more convenient to use polar coordinates $(r, \theta)$ than Cartesian ones, with $\xi=r \cos (\theta)$ and $\eta=r \sin (\theta)$. For a point-like source located in the direction $\alpha_{0}=\rho_{0} \cos \left(\varphi_{0}\right)$ and $\beta_{0}=\rho_{0} \sin \left(\varphi_{0}\right)$ on the sky, the phasor $\exp \left(\frac{-2 i \pi}{\lambda}\left(\alpha_{0} \xi+\beta_{0} \eta\right)\right)$ accounting for the tilted incoming wavefront is written as $\exp \left(\frac{-2 i \pi}{\lambda} \rho_{0} r \cos \left(\theta-\varphi_{0}\right)\right)$.

When rotating the wavefronts around the optical axis by angles $\pm \psi / 2$, we must change $\cos \left(\theta-\varphi_{0}\right)$ for $\cos \left(\theta-\varphi_{0} \pm \frac{\psi}{2}\right)$ and $P(r, \theta)$ for $P\left(r, \theta \pm \frac{\psi}{2}\right)$. Denoting as $A_{1}$ and $A_{2}$ the waves passing through the arms 1 and 2 for simplicity's sake, we have

$$
\begin{aligned}
& A_{1}=\frac{1}{2} P(r, \theta-\psi / 2) \mathrm{e}^{-\mathrm{i} \frac{2 \pi}{\lambda} \rho_{0} r \cos \left(\theta-\varphi_{0}-\psi / 2\right)} \\
& A_{2}=\frac{1}{2} P(r, \theta+\psi / 2) \mathrm{e}^{-\mathrm{i} \frac{2 \pi}{\lambda} \rho_{0} r \cos \left(\theta-\varphi_{0}+\psi / 2\right)}
\end{aligned}
$$

Since we assume that the geometry of the aperture is insensitive to rotation, we have $P(r, \theta-\psi / 2) \equiv P(r, \theta+\psi / 2)$. The images of the aperture in the additive and subtractive output can be written as

$$
\begin{aligned}
& A^{+}=A_{2}+A_{1}=P(r, \theta) \cos \left(\omega_{1}\right) \mathrm{e}^{-\mathrm{i} \omega_{2}} \\
& A^{-}=A_{2}-A_{1}=\mathrm{i} P(r, \theta) \sin \left(\omega_{1}\right) \mathrm{e}^{-\mathrm{i} \omega_{2}}
\end{aligned}
$$

where

$$
\begin{aligned}
& \omega_{1}=\frac{2 \pi}{\lambda} \rho_{0} r \sin \left(\frac{\psi}{2}\right) \sin \left(\theta-\varphi_{0}\right), \\
& \omega_{2}=\frac{2 \pi}{\lambda} \rho_{0} r \cos \left(\frac{\psi}{2}\right) \cos \left(\theta-\varphi_{0}\right) .
\end{aligned}
$$

The intensities $I^{+}$and $I^{-}$in the additive and subtractive outputs, computed to be the modulus squared of the amplitudes, are complementary for each point in the aperture. For future use, we express the intensity $I^{-}$in the subtractive output as

$$
\begin{array}{r}
I_{\psi}^{-}\left(r, \theta, \rho_{0}, \varphi_{0}\right)=|P(r, \theta)|^{2} \sin ^{2}\left[\frac{2 \pi}{\lambda} \rho_{0} r \sin \left(\frac{\psi}{2}\right) \sin \left(\theta-\varphi_{0}\right)\right] \\
=\frac{1}{2}|P(r, \theta)|^{2}\left(1-\cos \left[\frac{4 \pi}{\lambda} \rho_{0} r \sin \left(\frac{\psi}{2}\right) \sin \left(\theta-\varphi_{0}\right)\right]\right) .
\end{array}
$$

For an on-axis point source, the intensity is zero for any value of $\psi$, all the light being rejected in the additive output, whereas an off-axis point source will be seen only when the angle $\psi$ departs enough from zero.

For the general case of an off-axis point source, the intensity distribution in the aperture is a fringe pattern of frequency $2 \rho_{0} \sin (\psi / 2) / \lambda$. For the symmetrical half-rotations used in the arms of the MZI, the fringes are displayed perpendicular to the direction $\varphi_{0}$ of the source. This can be easily demonstrated by inserting $\varphi_{0}=0$ into the above equation, as we did for the focal plane images. The off-axis being applied along the $\xi$-direction, the fringed intensity distribution can be written as 
$\sin ^{2}\left[\frac{2 \pi}{\lambda} \rho_{0} \sin \left(\frac{\psi}{2}\right) y\right]$, in which the orientation appears along the $\eta$ direction.

As $\psi$ increases, the fringes tighten, as shown in the two examples of Fig. 4. For its highest value, achieved at $\theta=\pi$, ARC produces the same pattern as AIC does. In the subtractive output of the MZI, ARC behaves as a perfect nulling coronagraph, since the full intensity of the on-axis point-source (e.g. the star) is rejected in the additive output. Intensities in the additive and subtractive outputs are complementary.

The light originating in an off-axis source (e.g. the planet) is separated into two parts in the outputs. The amount of light (of the planet) in the subtractive output is obtained by integrating the fringe pattern across the aperture. Normalizing this with respect to the collected intensity (unity multiplied by the surface $S$ of the aperture) and using the second form of Eq. (9), we have

$$
\begin{aligned}
& \Phi_{\psi}^{-}\left(\rho_{0}, \varphi_{0}\right)=\frac{1}{2 S}\left\{\iint|P(r, \theta)|^{2} r \mathrm{~d} r \mathrm{~d} \theta\right. \\
& \left.-\iint|P(r, \theta)|^{2} \cos \left[\frac{4 \pi}{\lambda} \rho_{0} r \sin \left(\frac{\psi}{2}\right) \sin \left(\theta-\varphi_{0}\right)\right] r \mathrm{~d} r \mathrm{~d} \theta\right\} .
\end{aligned}
$$

Assuming a clear (unapodized) aperture so that $|P(r, \theta)|^{2}=$ $P(r, \theta)$, we can recognize a cosine Fourier transform in the second term of the equation. In polar coordinates, the Fourier transform $\hat{f}(\rho, \varphi)$ of $f(r, \theta)$ can be written as:

$\hat{f}(\rho, \varphi)=\iint f(r, \theta) \mathrm{e}^{2 \mathrm{i} \pi \rho r \cos (\theta-\varphi)} r \mathrm{~d} r \mathrm{~d} \theta$,

an expression that can be simply derived by applying the transformation of variables from Cartesian to polar coordinates. The second integral in Eq. (10) can then be written as the real part of the Fourier transform, for the variables $\varrho=2 \rho_{0} \sin (\psi / 2) / \lambda$ and $\vartheta=\varphi_{0}+\pi / 2$. The $\pi / 2$ term is used there to transform the sine term into the cosine term present in the definition of the Fourier transform. We finally have

$\Phi_{\psi}^{-}\left(\rho_{0}, \varphi_{0}\right)=\frac{1}{2}\left(1-\frac{1}{S} \operatorname{Re}\left[\hat{P}\left(\frac{2 \rho_{0}}{\lambda} \sin \left(\frac{\psi}{2}\right), \varphi_{0}+\frac{\pi}{2}\right)\right]\right)$.

The term $\varphi_{0}+\pi / 2$ indicates that the fringes are orthogonal to the direction of the off-axis point source, as previously mentioned. The expression for the additive output $\Phi_{\psi}^{+}\left(\rho_{0}, \varphi_{0}\right)$ is obtained by substituting a plus sign with a minus sign in the expression.

For a full circular aperture of diameter $D$, the real part of the 2D Fourier transform is identical to the Hankel transform for the angular frequency $2 \rho_{0} \sin (\psi / 2)$. We have

$\Phi_{\psi}^{-}\left(\rho_{0}\right)=\frac{1}{2}-\frac{J_{1}\left(\frac{2 \pi}{\lambda} D \rho_{0} \sin (\psi / 2)\right)}{\frac{2 \pi}{\lambda} D \rho_{0} \sin (\psi / 2)}$.

For $\psi=\pi$, we recover the value of AIC given in Baudoz et al. (2000a).

The complementary transmissions in the additive and subtractive outputs for $\psi=\pi$ (AIC case) are given in Fig. 5 (top curves) as a function of $\rho_{0}$ in units of $\lambda / D$. For the useful coronagraphic subtractive output, the transmission oscillates around the value 0.5 outside $0.6 \lambda / D$. A maximum of flux of 0.56 is obtained for $0.81 \lambda / D$, with a local minimum of 0.46 for $1.34 \lambda / D$. This transmission is reported in the bottom curves of Fig. 5 and compared to the transmissions obtained for $\psi$ equal to $\pi / 4$ and $\pi / 8$. Making $\psi$ small produces a dilatation of the curve by a factor $1 / \sin (\psi / 2)$. The IWA becomes that of a telescope of diameter $D \sin (\psi / 2)$, while the flux and angular resolution outside the IWA remain those of the telescope of diameter $D$.
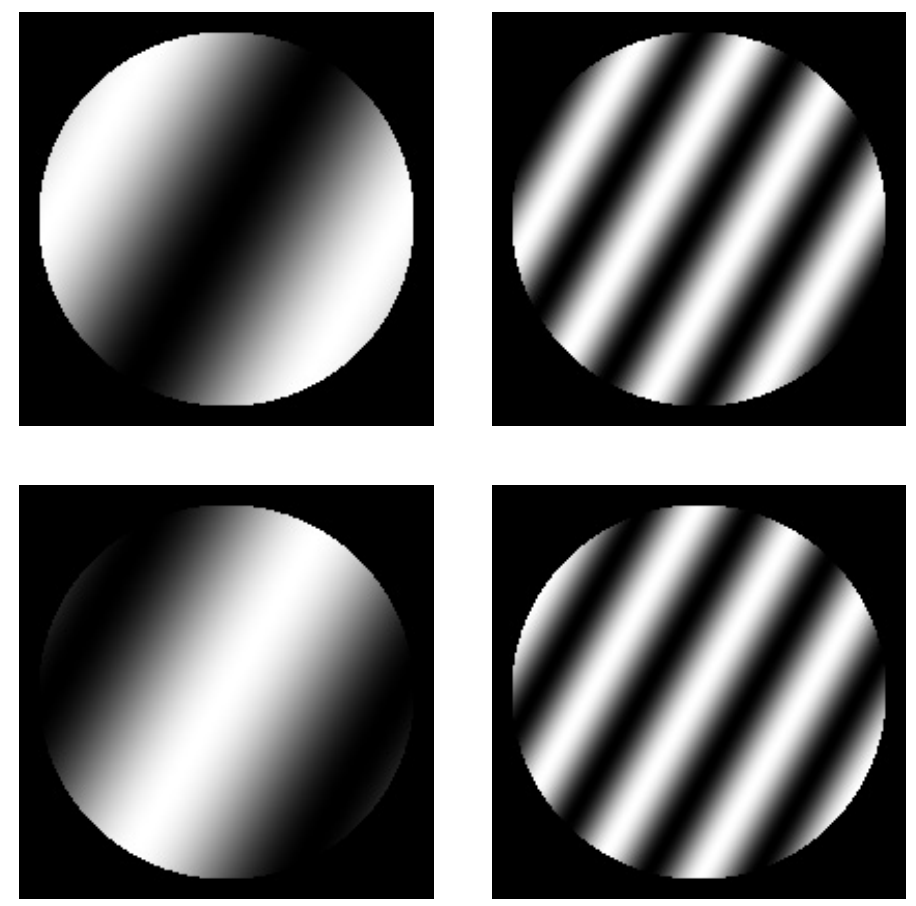

Fig. 4. Fringes in the aperture image (uniform disc) seen in the subtractive (top) and additive (bottom) outputs. Image observed for $\rho_{0}=$ $2 \lambda / D, \varphi_{0}=\pi / 4$ and $\psi$, equal to $\pi / 6$ (left) and $\pi$ (right, AIC case).

With knowledge of the angular distance of the exoplanet from the star, the angle $\psi$ may be tuned to use the coronagraph at its optimal value of transmission. For example, if we were to observe a solar-like system at $10 \mathrm{pc}$ in the visible at $0.6 \mu \mathrm{m}$, then we would set $\psi$ so that $D \sin (\psi / 2) \sim 1 \mathrm{~m}$. For an exo-Jupiter, about 5 times farther, the condition reduces to $D \sin (\psi / 2) \sim 0.2 \mathrm{~m}$. But the interest in small $\psi$ is mainly driven by our wish to reduce the stellar leakage, as we see below.

\section{Expressions for the leakage due to a star of finite diameter for a circular aperture}

\subsection{Expression for the leakage in the aperture plane}

For a circular aperture, it is possible to obtain a simple expression for the total leakage induced by a star of finite diameter $\Theta$. Normalizing the transmitted flux by $\pi \Theta^{2} / 4$ corresponding to the surface of the star, we have for the relative leakage

$$
\begin{aligned}
L_{\psi}^{-}(\Theta) & =\frac{4}{\pi \Theta^{2}} \times 2 \pi \int_{0}^{\Theta / 2} \rho \Phi_{\psi}^{-}(\rho) \mathrm{d} \rho \\
& =\frac{1}{2}+2 \lambda^{2} \frac{J_{0}(\pi \Theta D \sin (\psi / 2) / \lambda)-1}{\pi^{2} \Theta^{2} D^{2} \sin ^{2}(\psi / 2)} .
\end{aligned}
$$

Assuming $\Theta$ to be very small, a limited series expansion of the Bessel function makes it possible to approximate the leakage as

$L_{\psi}^{-}(\Theta) \simeq \frac{\pi^{2} \Theta^{2}}{32 \lambda^{2}} D^{2} \sin ^{2}\left(\frac{\psi}{2}\right)$

This expression is identical to that derived by Baudoz (1999), if $D \sin (\psi / 2)$ is substituted with $D$.

The leakage as an integrated quantity increases proportionally to the surface of the telescope. The larger the telescope, the worse the integrated leakage. As discussed for Eq. (13), we can use a small value of $\psi$ to compensate for the effect of a large 

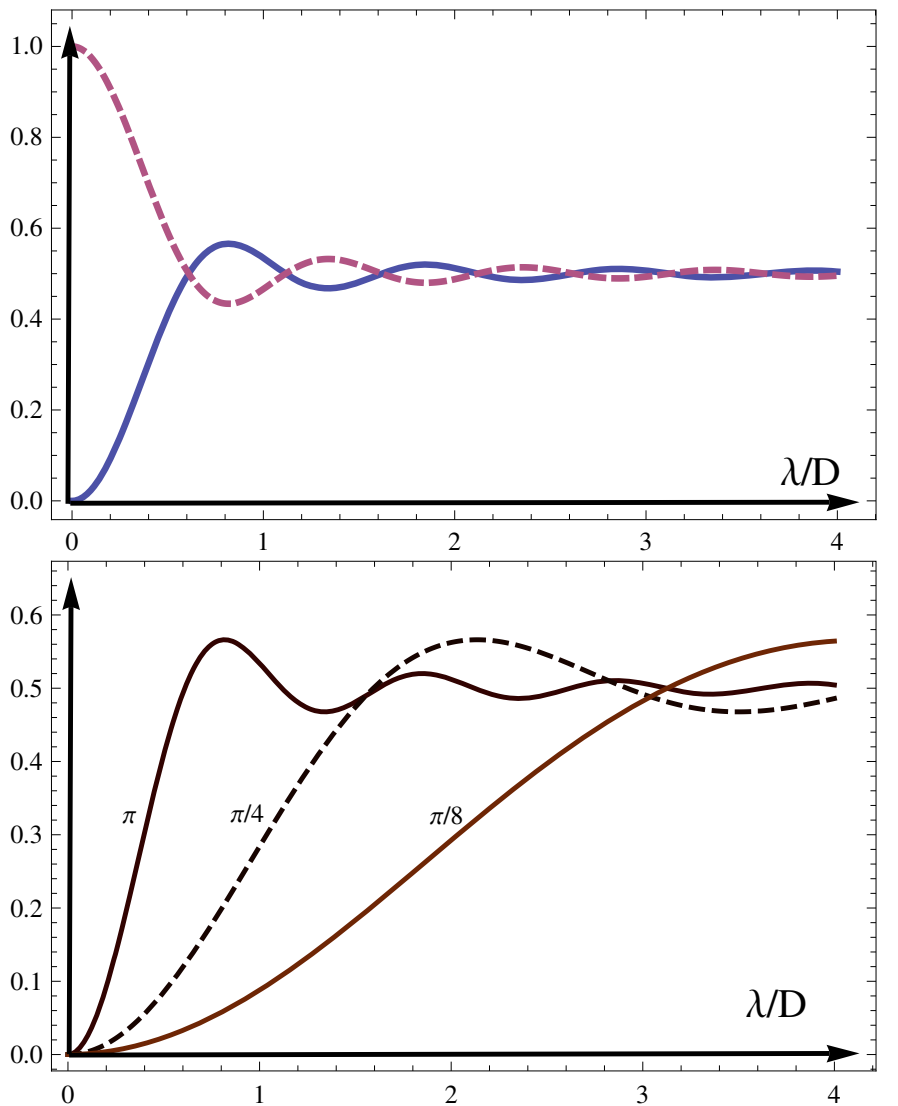

Fig. 5. Top: flux in the additive (dashed line) and subtractive (full line) outputs for $\psi=\pi$ (AIC case) as a function of the distance to the axis in units of $\lambda / D$. Bottom: flux in the subtractive outputs, for the values of $\psi$ equal to $\pi$ (AIC case), $\pi / 4$ and $\pi / 8$.

aperture. We consider again the example of the observation of a solar-like system at $10 \mathrm{pc}$. We have seen that the equivalent telescope sizes $(D \sin (\psi / 2))$ for the detection of an exo-Earth and an exo-Jupiter are 1-m and 0.2-m, respectively. If we express the diameter $D$ of the telescope in meters, then the gain in using ARC relative to AIC can be simply written as $D^{2}$ and $25 D^{2}$ for the exo-Earth and exo-Jupiter, respectively.

The gain in leakage reduction may even increase if a slightly smaller value of $\psi$ were used. There is, however, a limit for the integrated leakage reduction. It corresponds to that of the smallest telescope whose IWA is just large enough for it to be possible to observe the exoplanet. In the optimal case, using ARC with a small $\psi$ would cause a large telescope to produce the same leakage as a small one, and one may then be skeptical about the apparent need for a large telescope. The integrated leakage is not the relevant parameter that should be considered for planet detection. The relevant parameter is the residual intensity due to the star of finite diameter at the location of the planet, and to address this problem the use of a large telescope is mandatory, as we demonstrate in the next section.

\subsection{Expression for the leakage in the focal plane}

To derive the level of the residual intensity produced by the star of finite diameter at the location of the planet, we first need to develop a simplified workable expression for the intensity produced in the focal plane by a point source very close to the axis. We transpose the approach of Baudoz (1999) consisting of first writing an approximate expression for the amplitude in the aperture, and derive from it the intensity in the focal plane for very small values of $\rho_{0}$.

In Eq. (7), using for $\omega_{1}$ and $\omega_{2} \ll 1$ the approximation $\sin \left(\omega_{1}\right) \mathrm{e}^{\mathrm{i} \omega_{2}} \simeq \omega_{1}$, we have for the amplitude at the aperture

$A^{-}(r, \theta) \simeq \mathrm{i} P(r, \theta) \frac{2 \pi}{\lambda} \rho_{0} r \sin \left(\frac{\psi}{2}\right) \sin \left(\theta-\varphi_{0}\right)$.

The Fourier transform of this expression infers the amplitude in the focal plane, as shown in the Appendix. The approximate expression of the intensity for a point source at a position $\left(\rho_{0}, \varphi_{0}\right)$ that is very close to the axis can be written

$I(\rho, \varphi) \simeq \frac{\pi^{2} D^{4}}{4 \lambda^{2}} \sin ^{2}\left(\frac{\psi}{2}\right) \frac{J_{2}^{2}(\pi \rho D / \lambda)}{\rho^{2}} \rho_{0}^{2} \sin ^{2}\left(\varphi-\varphi_{0}\right)$.

To obtain the leakage in the focal plane, we have to sum incoherently all the contributions of the star of diameter $\Theta$ to obtain

$$
\begin{aligned}
I= & \frac{4}{\pi \Theta^{2}} \times \frac{\pi^{2} D^{4}}{4 \lambda^{2}} \sin ^{2}\left(\frac{\psi}{2}\right) \frac{J_{2}^{2}(\pi \rho D / \lambda)}{\rho^{2}} \\
& \times \int_{0}^{\Theta / 2} \rho_{0}^{3} \mathrm{~d} \rho_{0} \int_{0}^{2 \pi} \sin ^{2}\left(\varphi-\varphi_{0}\right) \mathrm{d} \varphi_{0} \\
= & \frac{\pi^{2} D^{4} \Theta^{2}}{64 \lambda^{2}} \sin ^{2}(\psi / 2) \frac{J_{2}^{2}(\pi \rho D / \lambda)}{\rho^{2}},
\end{aligned}
$$

an expression similar to what can be found in Baudoz (1999) by substituting $\Theta \sin (\psi / 2)$ with $\Theta$.

We can check the consistency of the result obtained here with the integrated leakage in the aperture plane. To make the expressions comparable, we first normalize the above expression of the leakage by the surface of the aperture and integrate it over the whole focal plane to obtain

$$
\begin{aligned}
L_{\psi}^{-}(\Theta) \simeq & \frac{4}{\pi D^{2}} \times \frac{\pi^{2} D^{4} \Theta^{2}}{64 \lambda^{2}} \sin ^{2}(\psi / 2) \\
& \times 2 \pi \int_{0}^{\infty} \frac{J_{2}^{2}(\pi \rho D / \lambda)}{\rho} \mathrm{d} \rho=\frac{\pi^{2} \Theta^{2} D^{2}}{32 \lambda^{2}} \sin ^{2}\left(\frac{\psi}{2}\right),
\end{aligned}
$$

where the result of Eq. (15) is retrieved. This is obtained from two completely different points of view, the first being the series approximation of a Bessel function once the final result is obtained, the second being an approximation of the wavefront at the aperture before the computation of its diffraction in the focal plane.

\subsection{Contrast and SNR considerations}

We can model an observation with ARC in the subtractive focal plane in terms of the twin-images of the planet we wish to detect superimposed on a background caused by the star leakage. Using Eqs. (5) and (18), we can write

$I(\alpha, \beta)=\frac{\pi^{2} D^{4} \Theta^{2}}{64 \lambda^{2}} \sin ^{2}\left(\frac{\psi}{2}\right) \frac{J_{2}^{2}(\pi \rho D / \lambda)}{\rho^{2}}+\varepsilon \times I_{\odot}^{-}(\alpha, \beta)$,

where $\varepsilon$ represents the ratio of the planet to star brightness.

We may use a simplified expression for Eq. (20) considering that the leakage is a smooth function over which we expect to see the planet. For that, the Bessel function may be approximated by its asymptotic form given by

$J_{2}(z) \simeq \sqrt{\frac{2}{\pi z}} \cos (z-3 \pi / 4) \leq \sqrt{\frac{2}{\pi z}}$ 

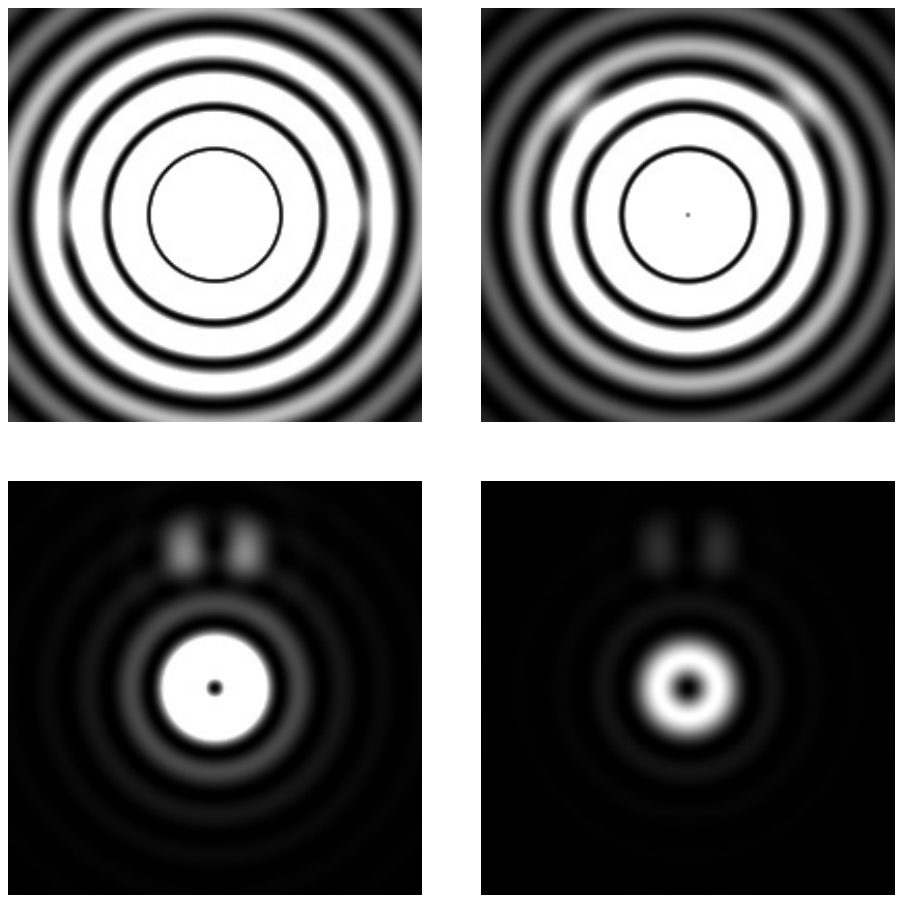

Fig. 6. Illustration of Eq. (20) showing the twin images of a planet $(\varepsilon=$ $10^{-7}, \rho_{0}=3.5 \lambda / D$ ) over the background caused by the leakage caused by the star of diameter $0.01 \lambda / D$. From top left to bottom right, $\psi$ values are $\pi, \pi / 2, \pi / 10$, and $\pi / 20$.

which ensures that the first term of Eq. (20) can be approximated by $D^{3} \Theta^{2} \sin ^{2}(\psi / 2) /\left(32 \lambda \rho^{3}\right)$, a rather slowly decreasing background (in $\left.\rho^{-3}\right)$. For $\rho_{0}>\lambda /(D \sin (\psi / 2))$, we can assume that the planet corresponds to two separated images. From Eq. (5), we can approximate the maximum intensity in one image of the planets to be $1 / 4$ of the nominal response, i.e. $\pi^{2} D^{4} /\left(64 \lambda^{2}\right)$. If we wish the amplitude of the planet to be larger than the background, we must assume that

$\varepsilon>\frac{2 \Theta^{2}}{\pi^{2} \rho_{0}^{3}} \frac{\lambda}{D} \sin ^{2}(\psi / 2)$.

This statement is pessimistic, since it is not in the background itself but in its fluctuations that the problem lies. Nevertheless, we consider again the solar-like system at $10 \mathrm{pc}$. We wish to detect a planet at the respective positions of Earth and Jupiter. We assume that we observe with a perfect 10-m spaceborn telescope operating in the visible, so that the resolution element $\lambda / D$ is about 0.01 arcsec. The diameter of the exo-Sun corresponds to $\Theta \simeq 0.001 \mathrm{arcsec}$, the exo-Earth is at $0.1 \mathrm{arcsec}$, and Jupiter is at 0.5 arcsec.

With $\psi=\pi$, to overcome the leakage at the distance corresponding to the Earth position, $\varepsilon$ should be greater than $2 \times 10^{-6}$, and $1.6 \times 10^{-8}$ at the position of Jupiter. This is not enough to see an exo-Earth, and makes it difficult to see an exo-Jupiter. We therefore reduce the value of $\psi$. Using the values already computed in round numbers, we can obtain $\sin (\psi / 2)=0.1$ for the exo-Earth and $\sin (\psi / 2)=0.02$ for the exo-Jupiter. The conditions are then $\varepsilon_{\mathrm{E}}>2 \times 10^{-8}$ for the exo-Earth and $\varepsilon_{\mathrm{J}}>6.4 \times 10^{-12}$ for the exo-Jupiter. The detection of an exo-Jupiter becomes certainly possible for ARC, and there remains little gain in detecting an exo-Earth, using either a slightly smaller $\psi$ value or a larger telescope.

The thoroughly relevant figure of merit in our context is the SNR pertaining to the extraction of the planet contribution
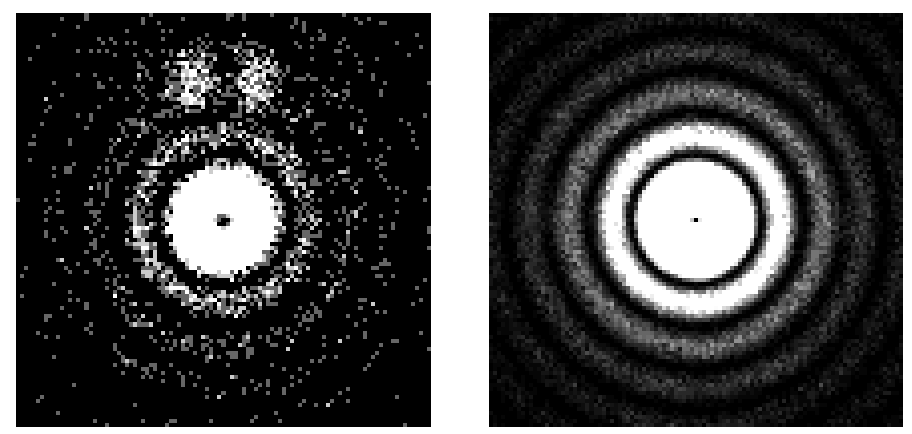

Fig. 7. Photon counting images of Fig. 6 for the $\psi$ values $\pi / 10$ (left) and $\pi$ (right). The number of photons in the planet is 500 and the leakage effect is clearly visible.

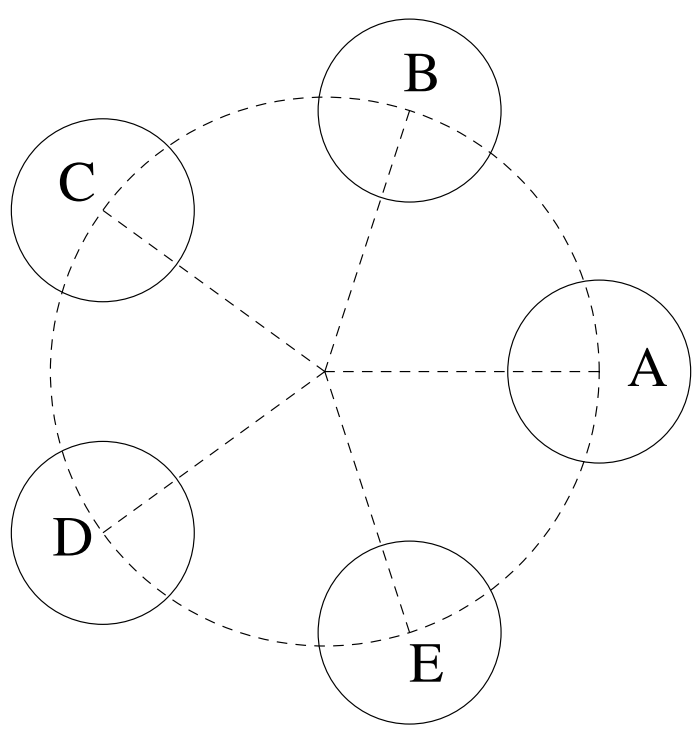

Fig. 8. Example of an ensemble of 5 telescopes in a pentagon configuration that can use ARC. The telescopes are set on a circle of diameter 3 times the telescope diameters.

against the noisy background. Following Aime (2005) and Rabbia et al. (1998), the SNR can be estimated to be the ratio of the number of photons in the image of the planet to the fluctuation in the number of photons in the background. The number of photons collected in the images of the planet is of the order of $k S / 2$, where $S$ is the surface of the telescope aperture and $k$ a factor that depends on the integration time, spectral bandwidth, and other experimental parameters. We note that the factor $k$ must be written so as to convert the collecting surface into a number of photons. The above factor $1 / 2$ is an approximation for the exact value, which depends on the planet distance and can be obtained using Fig. 5.

In the focal plane, the surface under the two images is $2 \times$ $\lambda^{2} / S$. The number of photons due to the leakage is

$k \frac{D^{3} \Theta^{2}}{32 \lambda \rho_{0}^{3}} \sin ^{2}(\psi / 2) \times \frac{8 \lambda^{2}}{\pi D^{2}}$,

and the SNR can then be approximately given by

$S N R \simeq \sqrt{k} \frac{\left(\pi D \rho_{0}\right)^{3 / 2}}{4 \sqrt{\lambda} \Theta \sin (\psi / 2)}$

The SNR increases as the inverse of $\sin (\psi / 2)$, which means that, for the example of the Solar-like system at $10 \mathrm{pc}$ observed by 

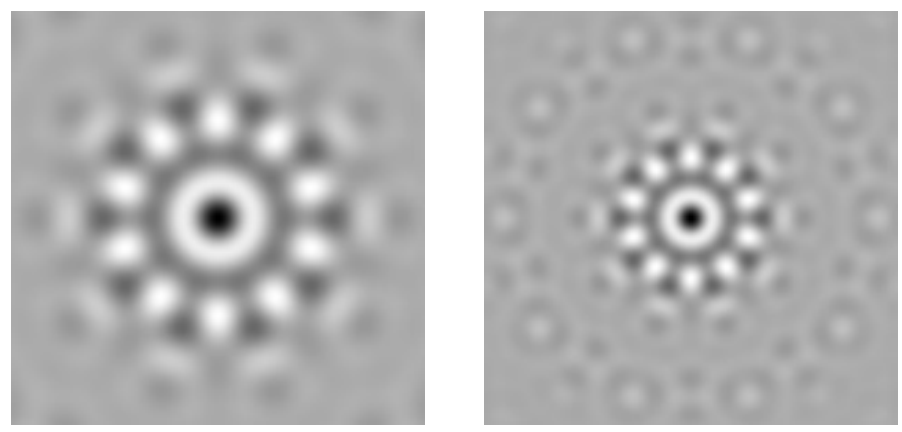

Fig. 9. On sky transmission for the pentagon configuration of Fig. 8 for the two rotations $\psi$ equal to $2 \pi / 5$ (left) and $4 \pi / 5$ (right). The figures are of the same scale, the apparent zoom effect being caused by the different rotation angles.

a $10-\mathrm{m}$ telescope in the visible, it equals a factor of 10 for an exo-Earth, and of 50 for an exo-Jupiter. The SNR increases as $D^{1.5}$ and the conclusion of a large D and a small $\psi$ derived for the contrast still holds for the SNR.

Examples of simulations obtained for various values of $\psi$ and illustrative values of $\Theta=0.01 \lambda / D$ and $\varepsilon=10^{-7}$ are shown in Fig. 6. In this example, the full expression given in Eq. (20) is used, showing the rings produced by the Bessel function. Since there is no noise in this representation, the twin images of the planet can be seen in the 4 images corresponding to $\psi$ values of $\pi, \pi / 2, \pi / 10$, and $\pi / 20$. The detection is optimal for $\psi=\pi / 10$. The effect of using a small $\psi$ value is more clearly visible in Fig. 7 for a simulated photon counting image of the focal plane of Fig. 6. The number of photons in the planet is about 500 in both cases. Owing to leakage, the number of photons in the image is about 9000 for $\psi=\pi / 10$ and 340000 for $\psi=\pi$.

\section{ARC used with diluted apertures}

As already mentioned, ARC can be used only if the aperture is invariant under a rotation by an angle $\psi$. For a single circular aperture, $\psi$ may take any value between 0 and $\pi$, the values between 0 and $-\pi$ duplicating these former ones. For diluted apertures, depending on their number and configuration, there may be a few values of $\psi$ satisfying the condition $P(r, \theta)=P(r, \theta-\psi)$. We note that a rotation of $-\psi$ provides the same result.

A basic configuration is a distribution of apertures equally spaced along a circle. The number of discrete values of $\psi$ varies with the number of apertures and are those (absolute value or modulo $\pi$ ) that can be used to reproduce after rotation the diluted pattern so as to superimpose any couple of individual apertures.

We begin with the basic 2-telescope Bracewell interferometer an example of which is the project FKSI (Danchi et al. 2003; Danchi \& Lopez 2007), where a single rotation $\psi=\pi$ allowed. For 3 apertures forming an equilateral triangle, there is still only one rotation fulfilling the requirement and corresponding to $\psi=2 \pi / 3$, since the other rotation $4 \pi / 3$ or $-2 \pi / 3$ provides the same result. For 4 apertures, there are 2 values, $\pi / 2$ and $\pi$. The number of different rotations for a regular polygonal configuration of $N$ apertures is the integer part of $N / 2$. An even number of apertures allows us to use the most resolving $\pi$ angle of rotation for $\psi$.

For illustration, we show in Fig. 8 a system of 5 apertures in a pentagon configuration, the apertures being denoted A, B,C,D and $\mathrm{E}$. There are 4 possible rotations that can superimpose $\mathrm{A}$ to $\mathrm{B}, \mathrm{C}, \mathrm{D}$ or $\mathrm{E}$, leading to the only two different angles of rotation $2 \pi / 5$ and $4 \pi / 5$, since only the absolute value of $\psi$ matters.
These two angles correspond to two different on-sky transmissions represented in Fig. 9 obtained using Eq. (12). These figures are 2D representations similar to Fig. 5, which represented a cut through the $2 \mathrm{D}$ transmission map for various $\psi$ values. For a circular aperture, this angle can take any value. For the pentagon configuration of a diluted array, the angle of rotation $2 \pi / 5$ is less resolving than the angle of rotation $4 \pi / 5$, but will ensure superior nulling because of star leakage considerations. The transmission peaks are close to 0.75 .

A detailed analysis of the application of the ARC technique to diluted apertures would require supplementary studies. The main point is that a null similar to the Bracewell interferometer can be obtained with many apertures by keeping the $\pi$ term of phase provided by the MZI. In other words, instead of insisting on challenging phase shift values (as $2 \pi / 5$ ) achievable only by means of dispersive plates (Mieremet et al. 2000; Rabbia et al. 2003) that are probably unstable in space conditions, the nulling process offered by ARC simply relies on $\pi$ phase shifts that are far easier to implement.

\section{Conclusion}

We have proposed a new coronagraphic concept that is similar in broad lines to AIC but takes advantage of the intrinsic $\pi$-phase shift providing the dark fringe of a MZI. In addition, ARC uses field rotators to transform the MZI into a rotation-shearing coronagraph. Two images are formed through the arms of the interferometer. If the center of rotation is set perfectly at the center of the stellar image, at the recombination outputs, the images of the star will be superimposed. The images of the planet will move along a circular path that depends on both its stellar distance and the angle of rotation.

Since the system uses a MZI, it provides two outputs, an additive and a subtractive output. Most of the equations that have been given in this paper are applicable to both outputs by modifying a minus sign into a plus sign, but the useful output is the subtractive one. The additive output, as demonstrated in this paper, will preferably be used to improve the pointing and the wavefront correction. When the angular separation of the twin images is 180 degrees, ARC offers the same theoretical capabilities as AIC, in particular the currently narrowest IWA of all coronagraphs. ARC makes it possible to optimize (maximize) the SNR by tuning the IWA, which is achieved by selecting the appropriate rotation angle and depends on the apparent diameter of the star and the planet-to-star separation. The close sensing range for ARC is intended to be of a few Airy radius (typically from 1 to $10 \lambda / D$ ), which provides support for a coronagraphic approach.

Two possible optical assemblies have been proposed, a MZI with field rotators (called ARC1), and a more compact out-ofplane folded MZI (called ARC2). This latter system may appear to be more elegant, but the basic ARC1 assembly will probably be far simpler to implement and to operate, as in the case of tuning the rotation angle $\psi$ with respect to the peculiar astronomical target. We propose this coronagraph to be optimal for the detection of exoplanets while we admit that it could not be used to image the environment of stars.

Several experimental and theoretical studies remain to be completed. A laboratory prototype of ARC1 is being developed in our institute, and for this initial version, relies on Dove prisms as field rotators, in a system similar to that proposed by Strojnik \& Paez (2007). These Dove prisms are convenient because the rotation is performed using a single optical component, but they are not the optimal solution when high rejection is required. The 
behavior of polarization and its influence on rejection is likely to be a critical issue. As has been shown here, the solution we envisage relies on the study conducted by Roddier et al. (1978), after adapting their system to ARC. These authors claimed that their phase-compensated roof-prisms should provide a perfect correction, but the question remains of whether their solution is capable of achieving in unpolarized light the ultra deep nulls required for exoplanet detection. The formalism they used for roof-prisms may be generalized to Dove prisms, but the effect of a system of mirrors may require a different approach. The system of Roddier et al. (1978) was used for variable angles of rotations, but we anticipate that the useful rotation angles for ARC will be either a very small angle, or an angle of $\pi$ to make ARC similar to AIC and complement it with an aperture apodization. In both situations, the residual effect of polarization will be minimized, but this is beyond the scope of this paper and will have to be checked by future theoretical and experimental studies.

The mathematical analysis has been performed by considering a perfect instrument operating outside the Earth's atmospheric turbulence and we have used rather simple models to derive the available contrast and SNR. It would also be interesting to study the behavior of ARC when extreme adaptive optics are used. We have again used very simplified models to derive the available contrast and SNR. The existence of twin images has not been used in our computation, and an additional elaborated data processing should take advantage of this point.

ARC remains a coronagraph with a $\Theta^{2}$ dependence on the leakage of starlight from a star that has a finite diameter $\Theta$, but this dependence can be written as $\alpha \times \Theta^{2}$, where $\alpha$ is proportional to $\sin ^{2}(\psi / 2)$. Using a small value of $\psi$ and a telescope of large diameter, ARC can in principle detect an exo-Earth, a performance that seems difficult to envision with coronagraphs using phase masks such as those of Roddier \& Roddier (1997), Rouan et al. (2000), and Aime et al. (2002), which are very efficient for an on-axis point source but strongly sensitive to star leakage. Coronagraphs using pupil or focal plane apodization, such as those of Soummer et al. (2003) and Kuchner \& Traub (2002), will be less sensitive to leakage with the drawback of a lower rejection or transmission efficiency.

The numerical simple computation we have given for a Solar-like system located at $10 \mathrm{pc}$ away from us has demonstrated that the detection of an exo-Jupiter may be feasible with a telescope of a few meters, but that a very large telescope (at least in the 10-m range) seems to be required to detect an exoEarth, due to stellar leakage. For a telescope of smaller diameter, such a coronagraph would not be efficient enough, and more complex solutions should be considered. One solution would be to use two interferometers in cascade to obtain fourth-order responses following the approach of Tavrov et al. (2008), to reduce the integrated leakage. Another solution would be to use an apodized aperture combined with ARC. First estimates for such a system (which will be presented in a forthcoming publication) indicate that this latter solution would not significantly reduce the integrated leakage, but apodization would contribute to reduce the stellar leakage at the level of the planet. A complex system would be to take advantage of the MZI by applying both the present technique and the phase apodization technique described in Carlotti et al. (2008).

We have very briefly presented the application of ARC to diluted apertures. The principal interest of the rotation-nuller is obviously that it just needs a $\pi$ phase shift regardless the number of apertures. The feasibility of this experiment will however strongly depend on the quality of the compensation for polarization that could possibly be implemented in that configuration.
The study has only addressed some preliminary steps and additional studies and developments, which we intend to conduct in the near future, are necessary.

Acknowledgements. We wish to thank our colleague Dr François Henault for his interesting comments. Thanks are also due to the anonymous referee for his careful and constructive analysis, especially for remarks concerning polarization leading us to improve the paper.

\section{Appendix A: Derivation of the approximate response in the focal plane}

We begin with the expression of $A^{-}(r, \theta)$ of Eq. (16). Assuming that $P(r, \theta)=P(r)$, the Fourier transform in polar coordinates $\hat{A^{-}}(\rho, \varphi)$ of $A^{-}(r, \theta)$ can be written as

$$
\begin{aligned}
\hat{A^{-}}(\rho, \varphi) & =\frac{1}{\lambda} \hat{P}\left(\frac{\rho}{\lambda}, \varphi\right) \simeq \mathrm{i} \frac{2 \pi}{\lambda^{2}} \rho_{0} \sin \left(\frac{\psi}{2}\right) \\
& \times \int_{0}^{\infty} r^{2} P(r) \int_{0}^{2 \pi} \sin \left(\theta-\varphi_{0}\right) \mathrm{e}^{-2 \mathrm{i} \pi \rho r \cos (\theta-\varphi) / \lambda} \mathrm{d} \theta \mathrm{d} r .
\end{aligned}
$$

We perform the change of variable $\theta^{\prime}=\theta-\varphi$ and develop the sine function $\sin \left(\theta^{\prime}+\varphi-\varphi_{0}\right)$ as

$\sin \left(\theta^{\prime}+\varphi-\varphi_{0}\right)=\cos \left(\theta^{\prime}\right) \sin \left(\varphi-\varphi_{0}\right)+\sin \left(\theta^{\prime}\right) \cos \left(\varphi-\varphi_{0}\right)$.(A.2)

We use the properties of parity for the integration that cancel the integral on $\sin \left(\theta^{\prime}\right)$ to obtain

$$
\begin{aligned}
\hat{A^{-}}(\rho, \varphi) \simeq & \mathrm{i} \frac{2 \pi}{\lambda^{2}} \rho_{0} \sin \left(\frac{\psi}{2}\right) \sin \left(\varphi-\varphi_{0}\right) \\
& \times \int_{0}^{D / 2} r^{2} \int_{0}^{2 \pi} \cos \left(\theta^{\prime}\right) \mathrm{e}^{-2 \mathrm{i} \pi \rho \cos \left(\theta^{\prime}\right) / \lambda} \mathrm{d} \theta^{\prime} \mathrm{d} r \\
= & -2 \pi \frac{2 \pi}{\lambda^{2}} \rho_{0} \sin \left(\frac{\psi}{2}\right) \sin \left(\varphi-\varphi_{0}\right) \int_{0}^{D / 2} r^{2} J_{1}(2 \pi \rho r / \lambda) \mathrm{d} r \\
= & -\frac{\pi D^{2}}{2 \lambda} \frac{\rho_{0}}{\rho} \sin \left(\frac{\psi}{2}\right) \sin \left(\varphi-\varphi_{0}\right) J_{2}(\pi \rho D / \lambda)
\end{aligned}
$$

from which Eq. (17) follows.

\section{References}

Aime, C. 2005, A\&A, 434, 785

Aime, C.. 2007, Comptes Rendus Physique, 8, 273

Aime, C., Carlotti, A., \& Ricort, G. 2007, Comptes Rendus Physique, 8, 961

Aime, C., Soummer, R., \& Ferrari, A. 2002, A\&A, 389, 334

Allouche, F., Glindemann, A., Aristidi, E., \& Vakili, F. 2009, A\&A, 500, 1277

Baudoz, P. 1999, Le coronographe interférentiel achromatique, Ph.D. Thesis, U. de Nice Sophia Antipolis

Baudoz, P., Rabbia, Y., \& Gay, J. 2000a, A\&AS, 141, 319

Baudoz, P., Rabbia, Y., Gay, J., et al. 2000b, A\&AS, 145, 341

Bracewell, R. N. 1978, Nature, 274, 780

Carlotti, A., Ricort, G., Aime, C., El Azhari, Y., \& Soummer, R. 2008, A\&A, 477, 329

Carlotti, A., Ricort, G., \& Aime, C. 2009, A\&A, 504, 663

Chauvin, G., Lagrange, A., Dumas, C., et al. 2004, A\&A, 425, L29

Danchi, W. C., \& Lopez, B. 2007, Comptes Rendus Physique, 8, 396

Danchi, W. C., Allen, R. J., Benford, D. J., et al. 2003, in Earths: DARWIN/TPF and the Search for Extrasolar Terrestrial Planets, ed. M. Fridlund, T. Henning, \& H. Lacoste, ESA SP, 539, 83

Gay, J., \& Rabbia, Y. 1996, Acad. Sci. Paris C. R. Ser. B Sci. Phys., 322, 265

Gay, J., Rabbia, Y., \& Baudoz, P. 1997, Acad. Sci. Paris C. R. Ser. B Sci. Phys., 325,51

Goodman, J. W. 1985, Statistical Optics, ed. J. W. Goodman

Guyon, O. 2007, Comptes Rendus Physique, 8, 323

Kuchner, M. J., \& Traub, W. A. 2002, ApJ, 570, 900

Labeyrie, A. 2006, in EAS Publications Series, ed. M. Carbillet, A. Ferrari, \& C. Aime, 22, 189 
A\&A 517, A55 (2010)

Lyot, B. 1932, Z. Astrophys., 5, 73

Lyot, B. 1939, MNRAS, 99, 580

Marois, C., Macintosh, B., Barman, T., et al. 2008, Science, 322, 1348

Mawet, D., Serabyn, E., Liewer, K., et al. 2010, ApJ, 709, 53

Mieremet, A. L., Braat, J. J., Bokhove, H., \& Ravel, K. 2000, in SPIE Conf. Ser. 4006, ed. P. Léna \& A. Quirrenbach, 1035

Murakami, N., et al. 2008, PASP, 120, 1112

Rabbia, Y., Baudoz, P., \& Gay, J. 1998, in The Next Generation Space Telescope: Science Drivers and Technological Challenges, ed. B. Kaldeich, LIA Colloq., 34, ESA SP, 429, 279

Rabbia, Y., Gay, J., Rivet, J.-P., \& Schneider, J.-L. 2003, in GENIE - DARWIN

Workshop - Hunting for Planets, ESA SP, 522

Ridgway, S. T. 2004, in EAS Publ. Ser. 12, ed. C. Aime, \& R. Soummer, 49
Roddier, C., \& Roddier, F. 1983, ApJ, 270, L23

Roddier, F., \& Roddier, C. 1997, PASP, 109, 815

Roddier, F., Roddier, C., \& Demarcq, J. 1978, J. Opt., 9, 145

Roddier, F., Roddier, C., Petrov, R., et al. 1986, ApJ, 305, L77

Rouan, D., Riaud, P., Boccaletti, A., Clénet, Y., \& Labeyrie, A. 2000, PASP, 112, 1479

Soummer, R., Aime, C., \& Falloon, P. E. 2003, A\&A, 397, 1161

Strojnik, M., \& Paez, G. 2007, Infrared Physics and Technology, 49, 312

Swartzlander, Jr., G. A., Ford, E. L., Abdul-Malik, R. S., et al. 2008, Opt. Express, 16, 10200

Tavrov, A. V., Nishikawa, J., Tamura, M., et al. 2008, Appl. Opt., 47, 4915

Traub, W. A. 1988, in European Southern Observatory Astrophysics Symposia, ed. F. Merkle, 29, 1029 